\title{
Bax-inhibitor-1 knockdown phenotypes are suppressed by Buffy and exacerbate degeneration in a Drosophila model of Parkinson disease
}

\author{
P Githure M'Angale ${ }^{1}$ ， Brian E Staveley ${ }^{\text {Corresp. } 1}$ \\ ${ }^{1}$ Department of Biology, Memorial University of Newfoundland, St. John's, Newfoundland \& Labrador, Canada \\ Corresponding Author: Brian E Staveley \\ Email address: bestave@mun.ca
}

Background. Bax inhibitor-1 (BI-1) is an evolutionarily conserved cytoprotective transmembrane protein, that acts as a suppressor of Bax-induced apoptosis by regulation of endoplasmic reticulum (ER) stress-induced cell death. We knocked down BI-1 in the sensitive dopa decarboxylase (Ddc) expressing neurons of Drosophila melanogaster to investigate its neuroprotective functions. We additionally sought to rescue the $\mathrm{BI}$-1-induced phenotypes by co-expression with the pro-survival Buffy and determined the effect of Bl-1 knockdown on the neurodegenerative $\alpha$-synuclein-induced Parkinson disease model.

Methods. We used organismal assays to assess longevity of the flies to determine the effect of the altered expression of $B \mathrm{I}-1$ in the $D d c$-Gal4-expressing neurons by employing two RNAi transgenic fly lines. We measured the locomotor ability of these RNAi lines by computing the climbing indices of the climbing ability and compared them to a control line that expresses the lacZ transgene. Finally, we performed biometric analysis of the developing eye, where we counted the number of ommatidia and calculated the area of ommatidial disruption.

Results. The knockdown of BI-1 in these neurons was achieved under the direction of the Ddc-Gal4 transgene and resulted in shortened lifespan and precocious loss of locomotor ability. The co-expression of Buffy, the Drosophila anti-apoptotic Bcl-2 homologue, with BI-1-RNAi resulted in suppression of the reduced lifespan and impaired climbing ability. Expression of human $\alpha$-synuclein in Drosophila dopaminergic neurons results in neuronal degeneration, accompanied by the age-dependent loss in climbing ability. We exploited this neurotoxic system to investigate possible BI-1 neuroprotective function. The co-expression of $\alpha$-synuclein with BI-1-RNAi results in a slight decrease in lifespan coupled with an impairment in climbing ability. In supportive experiments, we employed the neuron-rich Drosophila compound eye to investigate subtle phenotypes that result from altered gene expression. The knockdown of Bl-1 in the Drosophila developing eye under the direction of the GMR-Gal4 transgene results in reduced ommatidia number and increased disruption of the ommatidial array. Similarly, the coexpression of BI-1-RNAi with Buffy results in the suppression of the eye phenotypes. The expression of $\alpha$ synuclein along with the knockdown of $B I-1$ resulted in reduction of ommatidia number and more disruption of the ommatidial array.

Conclusions. Knockdown of Bl-1 in the dopaminergic neurons of Drosophila results in a shortened lifespan and premature loss in climbing ability, phenotypes that appear to be strongly associated with models of Parkinson disease in Drosophila, and which are suppressed upon overexpression of Buffy and worsened by co-expression with $\alpha$-synuclein. This suggests that $B I-1$ is neuroprotective and its knockdown can be counteracted by the overexpression of the pro-survival $\mathrm{BCl}-2$ homologue. 
1 Bax-inhibitor-1 knockdown phenotypes are suppressed by Buffy and 2 exacerbate degeneration in a Drosophila model of Parkinson disease 3

4 P. Githure M’Angale and Brian E. Staveley§

5 Department of Biology, Memorial University of Newfoundland, St. John's, Newfoundland \&

6 Labrador, Canada, A1B 3X9

7

8 Corresponding author:

9 Brian Staveley

10232 Elizabeth Avenue, St. John's, Newfoundland \& Labrador, A1B 3X9, Canada

11 Email address: bestave@mun.ca 


\section{Abstract}

14 Background. Bax inhibitor-1 (BI-1) is an evolutionarily conserved cytoprotective

15 transmembrane protein, that acts as a suppressor of $B a x$-induced apoptosis by regulation of

16 endoplasmic reticulum (ER) stress-induced cell death. We knocked down BI-1 in the sensitive

17 dopa decarboxylase $(D d c)$ expressing neurons of Drosophila melanogaster to investigate its

neuroprotective functions. We additionally sought to rescue the BI-1-induced phenotypes by coexpression with the pro-survival Buffy and determined the effect of BI-1 knockdown on the neurodegenerative $\alpha$-synuclein-induced Parkinson disease model.

21 Methods. We used organismal assays to assess longevity of the flies to determine the effect of

22 the altered expression of $B I-1$ in the $D d c$-Gal4-expressing neurons by employing two RNAi

23 transgenic fly lines. We measured the locomotor ability of these RNAi lines by computing the

24 climbing indices of the climbing ability and compared them to a control line that expresses the

25 lac $Z$ transgene. Finally, we performed biometric analysis of the developing eye, where we

26 counted the number of ommatidia and calculated the area of ommatidial disruption.

27 Results. The knockdown of $B I-1$ in these neurons was achieved under the direction of the $D d c$ -

Gal4 transgene and resulted in shortened lifespan and precocious loss of locomotor ability. The co-expression of Buffy, the Drosophila anti-apoptotic Bcl-2 homologue, with BI-1-RNAi resulted in suppression of the reduced lifespan and impaired climbing ability. Expression of human $\alpha-$ synuclein in Drosophila dopaminergic neurons results in neuronal degeneration, accompanied by the age-dependent loss in climbing ability. We exploited this neurotoxic system to investigate possible BI-1 neuroprotective function. The co-expression of $\alpha$-synuclein with BI-1-RNAi results in a slight decrease in lifespan coupled with an impairment in climbing ability. In supportive experiments, we employed the neuron-rich Drosophila compound eye to investigate subtle phenotypes that result from altered gene expression. The knockdown of $B I-1$ in the Drosophila 
37 developing eye under the direction of the GMR-Gal4 transgene results in reduced ommatidia

38 number and increased disruption of the ommatidial array. Similarly, the co-expression of BI-1-

$39 R N A i$ with Buffy results in the suppression of the eye phenotypes. The expression of $\alpha$-synuclein

40 along with the knockdown of $B I-1$ resulted in reduction of ommatidia number and more

41 disruption of the ommatidial array.

42 Conclusions. Knockdown of $B I-1$ in the dopaminergic neurons of Drosophila results in a

43 shortened lifespan and premature loss in climbing ability, phenotypes that appear to be strongly

44 associated with models of Parkinson disease in Drosophila, and which are suppressed upon

45 overexpression of Buffy and worsened by co-expression with $\alpha$-synuclein. This suggests that BI-1

46 is neuroprotective and its knockdown can be counteracted by the overexpression of the pro-

47 survival $B c l-2$ homologue.

\section{Introduction}

49 Bax inhibitor-1 (BI-1) belongs to a diverse group of proteins, known as Transmembrane Bax

Inhibitor-1 Motif-containing (TMBIM) family (Henke et al. 2011; Li et al. 2014; Reimers et al.

51 2008; Rojas-Rivera \& Hetz 2015), that have been determined to be regulators of cell death. A

different nomenclature categorizes these proteins into the $L F G$ family, adopted from the family

member Lifeguard (Hu, Smith \& Goldberger 2009), which consists of at least six highly

conserved members in a wide range of organisms (Chae et al. 2003; Henke et al. 2011;

Huckelhoven 2004). These regulators of cell death, accomplish this role by the regulation of the

death receptor, modulation of the endoplasmic reticulum (ER) calcium homeostasis, ER stress

57 signalling pathways, autophagy, reactive oxygen species (ROS) production, cytosolic

58 acidification and, other cellular activities (Li et al. 2014; Rojas-Rivera \& Hetz 2015). The 
60 transcript (TEGT), and has been demonstrated to inhibit the effect of Bax-induced cell death

61 (Walter et al. 1995; Xu \& Reed 1998). Members of this protein family possess a BI-1-like

62 domain with six to seven transmembrane-spanning regions that are strongly associated with the

63 ER membranes (Carrara et al. 2012; Chae et al. 2004; Xu \& Reed 1998). BI-1 is highly

64 conserved across diverse species with eukaryotic homologues of $B I-1$ able to block Bax-induced

65 cell death when expressed in yeast (Chae et al. 2003), thus implying it regulates an evolutionarily

66 conserved cytoprotective pathway.

67 This protein though not structurally related to the $B$ cell lymphoma 2 family of proteins, forms a

68 complex with the pro-survival members Bcl-2 and Bcl- $\mathrm{X}_{\mathrm{L}}$ but not with Bax or Bak (Lisbona et

69 al. 2009; Xu \& Reed 1998). Therefore, it is likely the anti-apoptotic activity of BI-1/TMBIM6 is

70 mediated by interaction with pro-survival members of the $B c l-2$ family and acts downstream of

$71 \mathrm{Bcl}_{\mathrm{L}}$ (Xu et al. 2008). BI-1 deficient cells, that include neurons, are more sensitive to apoptosis

72 induced by ER stress and has been linked to the modulation of ER calcium homeostasis (Chae et

73 al. 2004; Dohm et al. 2006). This implicates BI-1 in a variety of human diseases that include

74 numerous cancers, obesity, liver diseases, autoimmune response, and diabetes (Kiviluoto et al.

75 2012; Li et al. 2014; Lisak et al. 2015; Robinson et al. 2011; Rojas-Rivera \& Hetz 2015).

76 Neuroprotective roles include, protection from oxygen-glucose deprivation, promotion of

77 neuronal proliferation and differentiation, and stress-induced protection (Dohm et al. 2006;

78 Hunsberger et al. 2011; Jeon et al. 2012; Krajewska et al. 2011). It regulates ROS production by;

79 modulation of unfolded protein response (UPR) induction in the ER (Lee et al. 2007),

80 suppression of mitochondria-mediated ROS production (Kim et al. 2012), reduction of

81 cytochrome P450 2E1 activity and regulation of the ER membrane lipid peroxidation (Kim et al. 
82 2009). BI-1 undoubtedly has significant cytoprotective roles and their abrogation lead to cellular

83 homeostatic dysfunction and disease.

84 Drosophila melanogaster appear to possess most of the TMBIM protein family homologues with

85 TMBIM6/BI-1 represented by BI-1/CG7188 (Attrill et al. 2015; Hu, Smith \& Goldberger 2009;

86 Rojas-Rivera \& Hetz 2015). Drosophila has been used as a model organism in the study of gene

87 expression and in human disease models, albeit with very promising results (Staveley 2015).

88 Several studies have used Drosophila to elucidate the importance of this protein in cellular

89 homeostasis; including functional conservation of this protein in evolutionarily diverse

90 organisms (Chae et al. 2003), BI-1 as a negative regulator of the ER stress sensor IRE1 $\alpha$ and its

91 role in the UPR (Lisbona et al. 2009), and its modulation of autophagy (Castillo et al. 2011).

92 Expression in the Ddc-Gal4-expressing neurons is the focus of our studies as they are very

93 sensitive to subtle differences in gene products and can be used to study ROS, ER stress,

94 apoptosis, autophagy and many other cellular processes. This is mainly because they degenerate

95 in an age-dependent manner and this degeneration manifests as deficiency in locomotor function

96 (Botella et al. 2009; Feany \& Bender 2000; Park, Schulz \& Lee 2007; Staveley 2015). The key

97 elements of the Drosophila model of Parkinson disease that utilizes the expression of a human $\alpha$ -

98 synuclein transgene to induce the PD-like symptoms (Feany \& Bender 2000); is its ability to

99 recapitulate some features of human PD that include, age-dependent loss of DA neurons that

100 manifest in age-dependent loss in locomotor function (Auluck et al. 2002; Botella et al. 2009;

101 Buttner et al. 2014; Feany \& Bender 2000; Kong et al. 2015; Staveley 2015; Wang et al. 2015;

102 Zhu et al. 2016). The spatio-temporal $U A S / G A L 4$ expression system (Brand \& Perrimon 1993),

103 and the availability of a plethora of promoters or enhancers of which TH-Gal4, elav-Gal4 and

$104 D d c-$ Gal4 are employed to model PD in flies. 
105 The $\mathrm{Bcl}-2$ family member homologues in Drosophila are limited to the single anti-apoptotic

106 Buffy and the pro-apoptotic Debcl (Colussi et al. 2000). In previous studies, the overexpression

107 of Buffy has been shown to confer survival advantages specifically in response to external stimuli

108 and in conditions of cellular stress (M'Angale \& Staveley 2016; Monserrate, Chen \& Brachmann

109 2012; Sevrioukov et al. 2007; Tanner et al. 2011). This point to an important role for this protein

110 in aspects of cell death. We investigated the outcome of the knockdown of BI-1 in Drosophila

111 neurons, and further determined whether there is an interaction with the anti-apoptotic Bcl-2

112 protein Buffy. We employed two different RNAi lines to determine the specificity of the effects

113 of knockdown of this gene and compared them to a control line. We further co-expressed $B I-1$ in

114 DA neurons along with $\alpha$-synuclein to investigate whether it possesses neuroprotective functions

115 by assessing the phenotypes that would result from knockdown of $B I-1$ and expression of $\alpha$ -

116 synuclein. Lastly, in supportive experiments we attempted to establish a role for BI-1 in the

117 Drosophila developing eye.

118 Materials and Methods

119 Bioinformatic analysis

120 The protein sequences were obtained from the National Center for Biotechnology Information

121 (NCBI; http://www.ncbi.nlm.nih.gov/protein/) and the domains were identified using the NCBI

122 Conserved Domain Database (CDD; http://www.ncbi.nlm.nih.gov/cdd) (Marchler-Bauer et al.

123 2015) and the Eukaryotic Linear Motif (ELM; http://elm.eu.org/) (Dinkel et al. 2016) which

124 focuses on annotation and detection of eukaryotic linear motifs (ELMs), also known as short

125 linear motifs (SLiMs). A multiple sequence alignment was done using Clustal Omega

126 (http://www.ebi.ac.uk/Tools/msa/clustalo/) (Goujon et al. 2010; Sievers et al. 2011) to show

127 conservation of the domains in the selected organisms. The prediction of the nuclear export

128 signal (NES) was by NetNES (http://www.cbs.dtu.dk/services/NetNES/) (la Cour et al. 2004). 
129 Further analysis of protein sequences was performed with Phyre2 (Kelley et al. 2015), a web

130 portal for protein modelling, prediction and analysis

131 (http://www.sbg.bio.ic.ac.uk/phyre2/html/page.cgi?id=index). The sub-cellular localisation was

132 performed by MultiLoc2 (Blum, Briesemeister \& Kohlbacher 2009) (https://abi.inf.uni-

133 tuebingen.de/Services/MultiLoc2). Transmembrane domains were further investigated and

134 identified using TMpred (Artimo et al. 2012), a program based on statistical analysis of TMbase

135 (http://www.ch.embnet.org/software/TMPRED_form.html).

\section{Drosophila media, stocks and derivative lines}

137 Stocks and crosses were maintained on standard cornmeal/molasses/yeast/agar media treated

138 with propionic acid and methylparaben to inhibit fungal growth. Stocks were kept at room

139 temperature while crosses and experiments for analysis of ageing and climbing ability were

140 carried out at $25^{\circ} \mathrm{C}$ while those for the eye analysis were performed at $29^{\circ} \mathrm{C}$.

141 The $P\{K K 100983 V I E-260 B$ stock hereby referred to as $U A S-B I-1-R N A i$ (1)

142 (http://stockcenter.vdrc.at/control/product/ VIEW_INDEX=0/ VIEW_SIZE=100/ product_id=

$143110358)$ and $w^{1118} ; P\{G D 1660\} v 37108$ hereby referred to as $U A S-B I-1-R N A i(2)$

144 (http://stockcenter.vdrc.at/control/product/ VIEW_INDEX=0/ VIEW_SIZE=100/ product_id=

145 37108) (Dietzl et al. 2007) were obtained from Vienna Drosophila Resource Center. Additional

146 information on the RNAi constructs can be obtained from http://www.flyrnai.org/up-torr/. The

$147 U A S-B u f f y$ (Quinn et al. 2003) was provided by Dr. L. Quinn (University of Melbourne), Ddc-

148 Gal4 flies (Li et al. 2000) by Dr. J. Hirsch (University of Virginia) and UAS- $\alpha$-synuclein (Feany

149 \& Bender 2000) by Dr. M. Feany (Harvard Medical School). GMR-Gal4 (Freeman 1996) and

$150 U A S$-lacZ flies were obtained from the Bloomington Drosophila Stock Center. 
151 The UAS- $\alpha$-synuclein/CyO; Ddc-Gal4/TM3; UAS- $\alpha$-synuclein/CyO; GMR-Gal4; UAS-

$152 B$ Buffy/CyO; Ddc-Gal4 and UAS-Buffy/CyO;GMR-Gal4 complex lines were used to overexpress

$153 \alpha$-synuclein or Buffy in neurons and the developing eye and were produced employing standard

154 homologous recombination and marker selection methods as previously described (M'Angale \&

155 Staveley 2016a; M'Angale \& Staveley 2016c). Gel electrophoresis was used to detect the

156 presence of PCR products.

\section{Ageing assay}

158 Several crosses of each genotype were performed and male flies collected upon eclosion and

159 assessed using a protocol previously described (M'Angale \& Staveley 2016; Todd \& Staveley

160 2012). For each genotype at least 200 flies were aged and scored every 2 days for the presence of

161 deceased adults (Staveley, Phillips \& Hilliker 1990). Survival data was analysed using GraphPad

162 Prism version 5.04, and curves were compared using the Log-rank (Mantel-Cox) test with

163 statistical significance determined at $95 \%$, at a $\mathrm{P} \leq 0.05$ with a Bonferroni correction.

164 Climbing assay

165 A cohort of the critical class male flies was collected upon eclosion and scored for their ability to

166 climb using a method that was previously described (Todd \& Staveley 2004). Climbing analysis

167 was performed using the GraphPad Prism version 5.04 and climbing curves were fitted using

168 non-linear regression and compared using $95 \%$ confidence interval with a P-value of 0.05 or less

169 being statistically significant.

\section{Scanning electron microscopy of the Drosophila eye}

171 Male flies were collected upon eclosion and aged for up to five days and then prepared for

172 scanning electron microscopy using a standard protocol as previously described (M'Angale \&

173 Staveley 2016). For each genotype, at least 10 different eye images were analysed using the

174 National Institutes of Health (NIH) ImageJ software (Schneider, Rasband \& Eliceiri 2012) and 
175 biometric analysis performed using GraphPad Prism version 5.04. Disruption area of the eye was

176 calculated as has been previously described (M'Angale \& Staveley 2012). Statistical

177 comparisons comprised one-way analyses of variance (ANOVA) and Dunnett's multiple

178 comparison tests. P-values less than 0.05 were considered significant.

\section{Results}

180 Drosophila $B I-1$ is closely related to the human homologue

181 The 245 amino acids Drosophila BI-1 isoform A has a $42 \%$ identity and 68\% similarity to the

182295 amino acids human isoform B. The Drosophila homologue has a BI-1 domain between

183 amino acids $21-223$ and the human version at $74-286$ (Figure 1) as determined by the NCBI

184 Conserved Domain Database (Marchler-Bauer et al. 2015). An alignment of the protein

185 sequences using Clustal Omega (Goujon et al. 2010; Sievers et al. 2011) shows high

186 conservation of the BI-1-like domain in the organisms analysed (Figure 1A). Six transmembrane

187 (TM) domains in both Drosophila and human BI-1 were identified using both Eukaryotic linear

188 motif (ELM) (Dinkel et al. 2013) and TMpred (Artimo et al. 2012). An analysis of membrane-

189 spanning domains by Phyre2 (Kelley et al. 2015) reveals seven TM domains (Figure 1B) in both

190 sequences that are highly identical in the cytoplasmic to intracellular orientation. An inhibitor of

191 apoptosis binding motif (IBM) at amino acids 1-5, an endoplasmic reticulum (ER) retention

192 motif at position 221-224, and binding motifs for Atg8 at position 212-224 and calmodulin at

193 amino acids 226-242 were identified by ELM. The presence of nuclear export signal (NES) was

194 detected in both Drosophila and human BI-1 using NetNES (la Cour et al. 2004) and only in

195 Drosophila using the ELM. The 3D modelling of these proteins using Phyre2 (Figure 1C) shows

196 a close similarity in the structure and the orientation of the transmembrane domains with the

197 image coloured by rainbow from the $\mathrm{N} \rightarrow \mathrm{C}$ terminus. 
198 Knockdown of $\boldsymbol{B I - 1}$ in DA neurons decreases lifespan and severely impairs locomotor 199 function

200 The expression of both $B I-1-R N A i$ lines in the Ddc-Gal4-expressing neurons results in decreased

201 lifespan and impaired locomotor function. The median lifespan for these flies was 54 days for $202 B I-1-R N A i(1)$ and 46 days for BI-1-RNAi (2) when compared to 70 days for the controls that 203 express the lacZ transgene as determined by the Log-rank (Mantel-Cox) test (Figure 2A). When

$204 B I-1$ is suppressed in these neurons, the flies develop an early onset impairment of locomotor 205 ability as determined by the nonlinear fitting of the climbing curves (Figure 2B). The 95\% CI for 206 the slope were 0.033 to 0.050 and 0.0175 to 0.0355 for the two RNAi lines respectively when 207 compared to 0.052 to 0.070 for the lac $Z$ control flies. These results appear to suggest a role for $208 B I-1$ in the protection of neurons in Drosophila.

209

210

211

212

213

214

215

216

217

218

219

220

221

\section{Buffy suppresses the BI-1-RNAi-induced phenotypes}

The directed overexpression of the pro-survival Bcl-2 homologue Buffy results in increased lifespan and improved climbing ability (M'Angale \& Staveley 2016). When Buffy is coexpressed with both $B I-1-R N A i$ lines in the Ddc-Gal4-expressing neurons, the results indicate an increased median lifespan of 70 days and 72 days respectively when compared to 74 days for Buffy co-expressed with lacZ control flies and 70 days for the $l a c Z$ flies, as determined by Logrank test (Figure $3 \mathrm{~A}$ ). The climbing ability of the $B I-1-R N A i$ flies was not significantly different from the Buffy co-expressed with lacZ controls as determined by comparison of the BI-1-RNAi climbing curves (Figure 3B) with the control curve. The 95\% CI for the slope of BI-1-RNAi (1) was 0.0340 to 0.057 and that of $B I-1-R N A i$ (2) was 0.040 to 0.061 when compared to 0.035 to 0.050 and 0.052 to 0.070 for the controls. Taken together these results suggest a pro-survival role for $B I-1$; as the phenotypes induced by its knockdown are significantly counteracted by the prosurvival Bcl-2 homologue Buffy. 
222 Knockdown of $\boldsymbol{B I}-1$ with the expression of $\boldsymbol{\alpha}$-synuclein slightly alters phenotypes

223 The expression of $\alpha$-synuclein in dopaminergic neurons results in impaired locomotor function

224 that is attributed to cellular toxicity. The co-expression of $B I-1-R N A i$ along with $\alpha$-synuclein in

225 the Ddc-Gal4-expressing neurons, slightly exacerbated the reduced survival and the loss in

226 climbing ability observed with the expression of $\alpha$-synuclein. The median lifespan was 52 days

227 and 54 days for flies that express BI-1-RNAi along with $\alpha$-synuclein compared to 58 days for

228 controls that co-express $\alpha$-synuclein along with the lac $Z$ transgene and 70 days for flies that

229 express the benign lacZ transgene (Figure 4A) as determined by Log rank test with $p<0.001$. A

230 comparison of the climbing curves by nonlinear fitting at $95 \%$ CI revealed they were

231 significantly different (Figure 4B), with a CI of 0.038 to 0.049 for BI-1-RNAi (1) and 0.025 to

2320.033 for BI-1-RNAi (2) co-expressed along with $\alpha$-synuclein and compared to 0.052 to 0.069 for

233 the $\alpha$-synuclein co-expressed with lacZ control flies. This implies that the knockdown of BI-1 in

234 the $D d c$-Gal4-expressing neurons abrogates its cytoprotective function and enhances the $\alpha$ -

235 synuclein-induced phenotypes.

236 Knockdown of $\boldsymbol{B I}-1$ in the eye decreases ommatidia number and increases degeneration, 237 phenotypes that are rescued upon Buffy overexpression

238 The directed knockdown of BI-1 in the Drosophila developing eye using the GMR-Gal4

239 transgene resulted in eyes with decreased number of ommatidia and a higher disruption of the

240 ommatidial array in both the RNAi lines that were tested (Figure $4 \mathrm{~A}-\mathrm{C}$ and $4 \mathrm{~J}$ ) as determined

241 by a one-way analysis of variance with a p value less than 0.0001 . Co-expression of both BI-1-

$242 R N A i$ lines with Buffy restored the mean number of ommatidia to control levels as determined by

243 a one-way analysis of variance with $p=0.2439$ and 0.2342 . The percentage disruption was

244 significantly different from the control flies (Figure 4D - F and 4K). Taken together, these

245 results suggest that BI-1 may play a pro-survival role in the development of the Drosophila eye 
246 and that Buffy suppresses the developmental eye defects that result from the knockdown of BI-1.

247 The knockdown of BI-1 along with $\alpha$-synuclein expression resulted in a significant decrease in

248 the number of ommatidia or increase in percentage disruption of the eye as determined by a one-

249 way analysis of variance with a p value less than 0.0001 in both instances (Figure $4 \mathrm{G}-\mathrm{I}$ and

250 4L). The number of ommatidia and percentage of disruption was worse than with either $\alpha$ -

251 synuclein expression or $B I-1$ knockdown. This indicates that the knockdown of $B I-1$ enhances

252 the $\alpha$-synuclein-induced eye defects.

\section{Discussion}

254 The knockdown of BI-1 via stable inducible RNAi in the Ddc-Gal4-expressing neurons of

255 Drosophila results in decreased survival and impaired climbing ability over time. Although there

256 is no known homologue of Bax in Drosophila, the only pro-apoptotic $B c l-2$ homologue is Debcl

257 (Brachmann et al. 2000; Colussi et al. 2000; Igaki et al. 2000; Zhang et al. 2000), and has been

258 demonstrated to possess pro-apoptotic functions. The Drosophila BI-1 is able to block Bax-

259 induced cell death in yeast (Chae et al. 2003), and reduction of $B I-1$ function induces cell death

260 (Xu \& Reed 1998). These results suggest neuronal dysfunction may result from degeneration or

261 death when the function of $B I-1$ is reduced in the Ddc-Gal4-expressing neurons. The BI-1-

262 induced cell death could occur through interaction with pro-survival Bcl-2 proteins at the ER

263 membrane (Xu \& Reed 1998) and especially Bcl-2 and Bcl- $\mathrm{X}_{\mathrm{L}}$ in humans and possibly Buffy in

264 Drosophila. BI-1 seems to be involved in cellular functions that are protective to ER stress-

265 induced apoptosis (Chae et al. 2004). It seems to do this by the regulation of calcium ions (Lisak

266 et al. 2015; Xu et al. 2008) and ROS (Kim et al. 2009; Lee, Kim \& Chae 2012). BI-1 regulates

267 ER stress by controlling ER-generated ROS accumulation and stress linked to the unfolded

268 protein response. Therefore, the knockdown of this important ER stress regulator in the DA 
269 neurons would result in neuronal degeneration and death. The only pro-survival $B c l-2$

270 homologue in Drosophila is Buffy (Quinn et al. 2003) and the overexpression of Buffy is known

271 to confer survival advantages to cells under normal conditions and under conditions of stress

272 (Clavier et al. 2014; M'Angale \& Staveley 2016; M'Angale \& Staveley 2016b; M'Angale \&

273 Staveley 2016c; Monserrate, Chen \& Brachmann 2012; Quinn et al. 2003; Sevrioukov et al.

274 2007). The overexpression of Buffy along with the knockdown of BI-1 resulted in the

275 suppression of the BI-1-induced phenotypes. This Buffy action may be specific to its interaction

276 with $\mathrm{BI}-1$ or to its general pro-survival signalling pathways. The rescue of the $B I-1$-induced

277 phenotypes in both the $D d c$-Gal4-expressing neurons and in the developing eye may indicate a

278 pro-survival role for $B I-1$ in Drosophila, as the pro-survival action of Buffy can abrogate its

279 phenotypes.

280 The expression of human $\alpha$-synuclein in DA neurons of Drosophila results in impaired climbing

281 ability (Feany \& Bender 2000), similar to what is observed in reduced BI- 1 function. The

282 expression of $\alpha$-synuclein along with the reduction of BI-1 activity significantly altered the

283 impaired locomotor ability observed. The age-dependent reduction of climbing ability could be a

284 result of BI-1-induced apoptosis coupled with neurotoxicity that result from $\alpha$-synuclein

285 accumulation and the subsequent dysfunction of cellular mechanisms. Although we observed the

286 enhancement of the $\alpha$-synuclein-induced phenotypes by the knock down of $B I-1$, marked by the

287 reduction in longevity and a precocious loss in climbing ability, the $B I-1$-induced phenotypes

288 were hardly altered by the expression of $\alpha$-synuclein. This observation may exclude the

289 involvement of $\alpha$-synuclein in the BI-1 pathway as its expression does not enhance the BI-1

290 phenotypes, and the observed phenotypes may be because of the knockdown of BI-1. All the

291 same, it appears that the presence of either of the mechanisms, vis a vis $B I-1$-induced apoptosis 
292 or $\alpha$-synuclein aggregation neurotoxicity, confers a great disadvantage to Ddc-Gal4-expressing 293 neurons.

294 The suppression of $B I-1$ in the Drosophila eye under the direction of the GMR-Gal4 transgene 295 results in a lower ommatidia number when compared to the control. BI-1 is an apoptosis 296 suppressor gene and the down-regulation of its protein product results in programmed cell death

297 (Li et al. 2014). The reduction in the ommatidia number observed is mainly due to the fusion of 298 ommatidia and the resulting ommatidia disarray. The knockdown of BI- 1 in the Drosophila eye 299 seems to exacerbate the Gal4-induced apoptosis that manifests as roughened eye phenotype 300 (Kramer \& Staveley 2003). The co-expression of the Bcl-2 pro-cell survival homologue Buffy 301 with $B I-1-R N A i$ results in the suppression of the phenotype, with the number of ommatidia and 302 the roughened eye restored to control levels. Buffy seems to ameliorate this phenotype and it is 303 possibly via a general action on survival signals or an interaction with BI-1.

304 The expression of $\alpha$-synuclein in the Drosophila eye results in reduced ommatidia and a highly 305 disrupted ommatidial array (Feany \& Bender 2000). This $\alpha$-synuclein-induced developmental 306 eye defects model is a viable system to show the effects of altered gene expression and its role in 307 neuroprotection. The co-expression of $\alpha$-synuclein with $B I-1-R N A i$ in the Drosophila eye resulted 308 in decreased ommatidia number and a highly disrupted ommatidial array when compared to the 309 control that expresses $\alpha$-synuclein. The number of ommatidia decreased further when $\alpha$-synuclein 310 was co-expressed with $B I-1-R N A i$. Additionally, the degree of disruption of the ommatidial array

311 was also increased. Though it did not appear to be additive in nature, it seems that the

312 combination of the expression of the neurotoxic $\alpha$-synuclein and the knockdown of the activity

313 of the anti-apoptotic BI-1 results in a worsening of the roughened eye phenotype. The

314 accumulation of $\alpha$-synuclein has been implicated in breakdown of cellular homeostasis that 
315 include apoptosis, ROS production, and autophagy (Chinta et al. 2010). The knockdown of BI-1

316 disrupts regulation of similar mechanisms as those implicated in $\alpha$-synuclein-induced

317 neurotoxicity that include apoptosis, autophagy and ROS production (Li et al. 2014). It therefore,

318 follows that the combined action of $\alpha$-synuclein expression and BI-1 knockdown worsened the

319 phenotypes that result from either $\alpha$-synuclein expression or BI-1 knockdown.

320

321

322

323

324

325

326

327

328

329

330

331

332

333

334

335

336

337

338

339

340

341

342

343

344

345

346

\section{Conclusions}

The knockdown of BI-I in the Ddc-Gal4-expressing neurons of Drosophila results in reduction

in lifespan and an age-dependent loss in climbing ability, phenotypes that are strongly associated

with the degeneration and loss of dopaminergic neurons. The co-expression of the pro-survival

Buffy with BI-1-RNAi results in the rescue of the phenotypes observed, it is possible that Buffy

and BI-1 participate in cellular pathways that promote anti-apoptosis. Finally, BI-1 appears to be

neuroprotective as its knockdown along with $\alpha$-synuclein expression result in enhanced

phenotypes.

\section{References}

Artimo P, Jonnalagedda M, Arnold K, Baratin D, Csardi G, de Castro E, Duvaud S, Flegel V, Fortier A, Gasteiger E, Grosdidier A, Hernandez C, Ioannidis V, Kuznetsov D, Liechti R, Moretti S, Mostaguir K, Redaschi N, Rossier G, Xenarios I, Stockinger H. 2012. ExPASy: SIB bioinformatics resource portal. Nucleic acids research 40: W597-W603.

Attrill H, Falls K, Goodman JL, Millburn GH, Antonazzo G, Rey AJ, Marygold SJ, consortium F. 2015. FlyBase: establishing a Gene Group resource for Drosophila melanogaster. Nucleic acids research.

Auluck PK, Chan HY, Trojanowski JQ, Lee VM, Bonini NM. 2002. Chaperone suppression of alpha-synuclein toxicity in a Drosophila model for Parkinson's disease. Science 295: 865868.

Blum T, Briesemeister S, Kohlbacher O. 2009. MultiLoc2: integrating phylogeny and Gene Ontology terms improves subcellular protein localization prediction. BMC Bioinformatics 10: 274.

Botella JAA, Bayersdorfer F, Gmeiner F, Schneuwly S. 2009. Modelling Parkinson's disease in Drosophila. Neuromolecular Medicine 11: 268-280.

Brachmann CB, Jassim OW, Wachsmuth BD, Cagan RL. 2000. The Drosophila bcl-2 family member dBorg-1 functions in the apoptotic response to UV-irradiation. Current Biology 10: $547-550$. 
347

348

349

350

351

352

353

354

355

356

357

358

359

360

361

362

363

364

365

366

367

368

369

370

371

372

373

374

375

376

377

378

379

380

381

382

383

384

385

386

387

388

389

390

391

392

Brand AH, Perrimon N. 1993. Targeted gene expression as a means of altering cell fates and generating dominant phenotypes. Development 118: 401-415.

Buttner S, Broeskamp F, Sommer C, Markaki M, Habernig L, Alavian-Ghavanini A, CarmonaGutierrez D, Eisenberg T, Michael E, Kroemer G, Tavernarakis N, Sigrist SJ, Madeo F. 2014. Spermidine protects against alpha-synuclein neurotoxicity. Cell Cycle 13: 39033908.

Carrara G, Saraiva N, Gubser C, Johnson BF, Smith GL. 2012. Six-transmembrane topology for Golgi anti-apoptotic protein (GAAP) and Bax inhibitor 1 (BI-1) provides model for the transmembrane Bax inhibitor-containing motif (TMBIM) family. The Journal of biological chemistry 287: 15896-15905.

Castillo K, Rojas-Rivera D, Lisbona F, Caballero B, Nassif M, Court FA, Schuck S, Ibar C, Walter P, Sierralta J, Glavic A, Hetz C. 2011. BAX inhibitor-1 regulates autophagy by controlling the IRE1alpha branch of the unfolded protein response. EMBO Journal 30: 4465-4478.

Chae H-J, Ke N, Kim H-R, Chen S, Godzik A, Dickman M, Reed JC. 2003. Evolutionarily conserved cytoprotection provided by Bax Inhibitor-1 homologs from animals, plants, and yeast. Gene 323: 101-113.

Chae HJ, Kim HR, Xu C, Bailly-Maitre B, Krajewska M, Krajewski S, Banares S, Cui J, Digicaylioglu M, Ke N, Kitada S, Monosov E, Thomas M, Kress CL, Babendure JR, Tsien RY, Lipton SA, Reed JC. 2004. BI-1 regulates an apoptosis pathway linked to endoplasmic reticulum stress. Molecular Cell 15: 355-366.

Chinta SJ, Mallajosyula JK, Rane A, Andersen JK. 2010. Mitochondrial alpha-synuclein accumulation impairs complex I function in dopaminergic neurons and results in increased mitophagy in vivo. Neuroscience Letters 486: 235-239.

Clavier A, Baillet A, Rincheval-Arnold A, Coleno-Costes A, Lasbleiz C, Mignotte B, Guenal I. 2014. The pro-apoptotic activity of Drosophila Rbf1 involves dE2F2-dependent downregulation of diap1 and buffy mRNA. Cell death \& disease 5: e1405.

Colussi PA, Quinn LM, Huang DC, Coombe M, Read SH, Richardson H, Kumar S. 2000. Debcl, a proapoptotic Bcl-2 homologue, is a component of the Drosophila melanogaster cell death machinery. The Journal of cell biology 148: 703-714.

Dietzl G, Chen D, Schnorrer F, Su K-CC, Barinova Y, Fellner M, Gasser B, Kinsey K, Oppel S, Scheiblauer S, Couto A, Marra V, Keleman K, Dickson BJ. 2007. A genome-wide transgenic RNAi library for conditional gene inactivation in Drosophila. Nature 448: 151-156.

Dinkel H, Van Roey K, Michael S, Davey NE, Weatheritt RJ, Born D, Speck T, Krüger D, Grebnev G, Kubań M, Strumillo M, Uyar B, Budd A, Altenberg B, Seiler M, Chemes LB, Glavina J, Sánchez IE, Diella F, Gibson TJ. 2013. The eukaryotic linear motif resource ELM: 10 years and counting. Nucleic acids research.

Dinkel H, Van Roey K, Michael S, Kumar M, Uyar B, Altenberg B, Milchevskaya V, Schneider M, Kuhn H, Behrendt A, Dahl SL, Damerell V, Diebel S, Kalman S, Klein S, Knudsen AC, Mader C, Merrill S, Staudt A, Thiel V, Welti L, Davey NE, Diella F, Gibson TJ. 2016. ELM 2016-data update and new functionality of the eukaryotic linear motif resource. Nucleic acids research 44: D294-300.

Dohm CP, Siedenberg S, Liman J, Esposito A, Wouters FS, Reed JC, Bahr M, Kermer P. 2006. Bax inhibitor-1 protects neurons from oxygen-glucose deprivation. Journal of molecular neuroscience 29: 1-8. 
393

394

395

396

397

398

399

400

401

402

403

404

405

406

407

408

409

410

411

412

413

414

415

416

417

418

419

420

421

422

423

424

425

426

427

428

429

430

431

432

433

434

435

436

437

438

Feany MB, Bender WW. 2000. A Drosophila model of Parkinson's disease. Nature 404: 394398.

Freeman M. 1996. Reiterative use of the EGF receptor triggers differentiation of all cell types in the Drosophila eye. Cell 87: 651-660.

Goujon M, McWilliam H, Li W, Valentin F, Squizzato S, Paern J, Lopez R. 2010. A new bioinformatics analysis tools framework at EMBL-EBI. Nucleic acids research 38: W695-W699.

Henke N, Lisak DA, Schneider L, Habicht J, Pergande M, Methner A. 2011. The ancient cell death suppressor BAX inhibitor-1. Cell Calcium 50: 251-260.

Hu L, Smith TF, Goldberger G. 2009. LFG: a candidate apoptosis regulatory gene family. Apoptosis 14: 1255-1265.

Huckelhoven R. 2004. BAX Inhibitor-1, an ancient cell death suppressor in animals and plants with prokaryotic relatives. Apoptosis 9: 299-307.

Hunsberger JG, Machado-Vieira R, Austin DR, Zarate C, Chuang DM, Chen G, Reed JC, Manji HK. 2011. Bax inhibitor 1, a modulator of calcium homeostasis, confers affective resilience. Brain Research 1403: 19-27.

Igaki T, Kanuka H, Inohara N, Sawamoto K, Nunez G, Okano H, Miura M. 2000. Drob-1, a Drosophila member of the Bcl-2/CED-9 family that promotes cell death. Proceedings of the National Academy of Sciences of the United States of America 97: 662-667.

Jeon K, Lim H, Kim JH, Han D, Lee ER, Yang GM, Song MK, Kim JH, Cho SG. 2012. Bax inhibitor-1 enhances survival and neuronal differentiation of embryonic stem cells via differential regulation of mitogen-activated protein kinases activities. Biochimica et Biophysica Acta (BBA) - Bioenergetics 1823: 2190-2200.

Kelley LA, Mezulis S, Yates CM, Wass MN, Sternberg MJE. 2015. The Phyre2 web portal for protein modeling, prediction and analysis. Nature Protocols 10: 845-858.

Kim HR, Lee GH, Cho EY, Chae SW, Ahn T, Chae HJ. 2009. Bax inhibitor 1 regulates ERstress-induced ROS accumulation through the regulation of cytochrome P450 2E1. Journal of Cell Science 122: 1126-1133.

Kim JH, Lee ER, Jeon K, Choi HY, Lim H, Kim SJ, Chae HJ, Park SH, Kim S, Seo YR, Kim JH, Cho SG. 2012. Role of BI-1 (TEGT)-mediated ERK1/2 activation in mitochondriamediated apoptosis and splenomegaly in BI-1 transgenic mice. Biochimica et Biophysica Acta (BBA) - Bioenergetics 1823: 876-888.

Kiviluoto S, Schneider L, Luyten T, Vervliet T, Missiaen L, De Smedt H, Parys JB, Methner A, Bultynck G. 2012. Bax inhibitor-1 is a novel IP(3) receptor-interacting and -sensitizing protein. Cell death \& disease 3: e367.

Kong Y, Liang X, Liu L, Zhang D, Wan C, Gan Z, Yuan L. 2015. High Throughput Sequencing Identifies MicroRNAs Mediating alpha-Synuclein Toxicity by Targeting NeuroactiveLigand Receptor Interaction Pathway in Early Stage of Drosophila Parkinson's Disease Model. PLoS One 10: e0137432.

Krajewska M, Xu L, Xu W, Krajewski S, Kress CL, Cui J, Yang L, Irie F, Yamaguchi Y, Lipton SA, Reed JC. 2011. Endoplasmic reticulum protein BI-1 modulates unfolded protein response signaling and protects against stroke and traumatic brain injury. Brain Research 1370: 227-237.

Kramer JM, Staveley BE. 2003. GAL4 causes developmental defects and apoptosis when expressed in the developing eye of Drosophila melanogaster. Genetics and Molecular Research 2: 43-47. 
439

440

441

442

443

444

445

446

447

448

449

450

451

452

453

454

455

456

457

458

459

460

461

462

463

464

465

466

467

468

469

470

471

472

473

474

475

476

477

478

479

480

481

482 la Cour T, Kiemer L, Mølgaard A, Gupta R, Skriver K, Brunak S. 2004. Analysis and prediction of leucine-rich nuclear export signals. Protein Engineering Design and Selection 17: $527-$ 536.

Lee GH, Kim HK, Chae SW, Kim DS, Ha KC, Cuddy M, Kress C, Reed JC, Kim HR, Chae HJ. 2007. Bax inhibitor-1 regulates endoplasmic reticulum stress-associated reactive oxygen species and heme oxygenase-1 expression. The Journal of biological chemistry 282: $21618-21628$.

Lee GH, Kim HR, Chae HJ. 2012. Bax inhibitor-1 regulates the expression of P450 2E1 through enhanced lysosome activity. The international journal of biochemistry \& cell biology 44: 600-611.

Li B, Yadav RK, Jeong GS, Kim HR, Chae HJ. 2014. The characteristics of Bax inhibitor-1 and its related diseases. Current Molecular Medicine 14: 603-615.

Li H, Chaney S, Roberts IJ, Forte M, Hirsh J. 2000. Ectopic G-protein expression in dopamine and serotonin neurons blocks cocaine sensitization in Drosophila melanogaster. Current Biology 10: 211-214.

Lisak D, Schacht T, Gawlitza A, Albrecht P, Aktas O, Koop B, Gliem M, Hofstetter HH, Zanger K, Bultynck G, Parys JB, De Smedt H, Kindler T, Adams-Quack P, Hahn M, Waisman A, Reed JC, Hovelmeyer N, Methner A. 2015. BAX inhibitor-1 is a Ca channel critically important for immune cell function and survival. Cell Death and Differentiation.

Lisbona F, Rojas-Rivera D, Thielen P, Zamorano S, Todd D, Martinon F, Glavic A, Kress C, Lin JH, Walter P, Reed JC, Glimcher LH, Hetz C. 2009. BAX inhibitor-1 is a negative regulator of the ER stress sensor IRE1alpha. Molecular Cell 33: 679-691.

M'Angale PG, Staveley BE. 2012. Effects of $\alpha$-synuclein expression in the developing Drosophila eye. Drosophila Information Services 95: 85-89.

M'Angale PG, Staveley BE. 2016. The Bcl-2 homologue Buffy rescues alpha-synuclein-induced Parkinson disease-like phenotypes in Drosophila. BMC Neuroscience 17: 24.

M'Angale PG, Staveley BE. 2016a. Bcl-2 homologue Debcl enhances $\alpha$-synuclein-induced phenotypes in Drosophila. PeerJ 4: e2461.

M'Angale PG, Staveley BE. 2016b. The HtrA2 Drosophila model of Parkinson Disease is suppressed by the pro-survival Bcl-2 Buffy. Genome In Press.

M'Angale PG, Staveley BE. 2016c. Inhibition of Atg6 and Pi3K59F autophagy genes in neurons decreases lifespan and locomotor ability in Drosophila melanogaster. Genetics and Molecular Research 15: gmr15048953.

Marchler-Bauer A, Derbyshire MK, Gonzales NR, Lu S, Chitsaz F, Geer LY, Geer RC, He J, Gwadz M, Hurwitz DI, Lanczycki CJ, Lu F, Marchler GH, Song JS, Thanki N, Wang Z, Yamashita RA, Zhang D, Zheng C, Bryant SH. 2015. CDD: NCBI's conserved domain database. Nucleic acids research 43: D222-226.

Monserrate JP, Chen MY, Brachmann CB. 2012. Drosophila larvae lacking the bcl-2 gene, buffy, are sensitive to nutrient stress, maintain increased basal target of rapamycin (Tor) signaling and exhibit characteristics of altered basal energy metabolism. BMC Biol 10: 63.

Park SS, Schulz EM, Lee D. 2007. Disruption of dopamine homeostasis underlies selective neurodegeneration mediated by alpha-synuclein. European Journal of Neuroscience 26: 3104-3112. 
483

484

485

486

487

488

489

490

491

492

493

494

495

496

497

498

499

500

501

502

503

504

505

506

507

508

509

510

511

512

513

514

515

516

517

518

519

520

521

522

523

524

525

526

527

528

Quinn L, Coombe M, Mills K, Daish T, Colussi P, Kumar S, Richardson H. 2003. Buffy, a Drosophila Bcl-2 protein, has anti-apoptotic and cell cycle inhibitory functions. EMBO Journal 22: 3568-3579.

Reimers K, Choi CY, Bucan V, Vogt PM. 2008. The Bax Inhibitor-1 (BI-1) family in apoptosis and tumorigenesis. Current Molecular Medicine 8: 148-156.

Robinson KS, Clements A, Williams AC, Berger CN, Frankel G. 2011. Bax inhibitor 1 in apoptosis and disease. Oncogene 30: 2391-2400.

Rojas-Rivera D, Hetz C. 2015. TMBIM protein family: ancestral regulators of cell death. Oncogene 34: 269-280.

Schneider CA, Rasband WS, Eliceiri KW. 2012. NIH Image to ImageJ: 25 years of image analysis. Nature Methods 9: 671-675.

Sevrioukov EA, Burr J, Huang EW, Assi HH, Monserrate JP, Purves DC, Wu JN, Song EJ, Brachmann CB. 2007. Drosophila Bcl-2 proteins participate in stress-induced apoptosis, but are not required for normal development. Genesis 45: 184-193.

Sievers F, Wilm A, Dineen D, Gibson TJ, Karplus K, Li W, Lopez R, McWilliam H, Remmert M, Söding J, Thompson JD, Higgins DG. 2011. Fast, scalable generation of high-quality protein multiple sequence alignments using Clustal Omega. Molecular Systems Biology 7: 539 .

Staveley BE. 2015. Drosophila Models of Parkinson Disease. In: LeDoux MS, ed. Movement Disorders: Genetics and Models. Second ed: Elsevier Science, 345-354.

Staveley BE, Phillips JP, Hilliker AJ. 1990. Phenotypic consequences of copper-zinc superoxide dismutase overexpression in Drosophila melanogaster. Genome 33: 867-872.

Tanner EA, Blute TA, Brachmann CB, McCall K. 2011. Bcl-2 proteins and autophagy regulate mitochondrial dynamics during programmed cell death in the Drosophila ovary. Development 138: 327-338.

Todd AM, Staveley BE. 2004. Novel assay and analysis for measuring climbing ability in Drosophila. Drosophila Information Services 87: 101-107.

Todd AM, Staveley BE. 2012. Expression of Pink1 with alpha-synuclein in the dopaminergic neurons of Drosophila leads to increases in both lifespan and healthspan. Genet Mol Res 11: 1497-1502.

Walter L, Marynen P, Szpirer J, Levan G, Günther E. 1995. Identification of a novel conserved human gene, TEGT. Genomics 28: 301-304.

Wang B, Liu Q, Shan H, Xia C, Liu Z. 2015. Nrf2 inducer and cncC overexpression attenuates neurodegeneration due to alpha-synuclein in Drosophila. Biochemistry and Cell Biology 93: 351-358.

$\mathrm{Xu}$ C, Xu W, Palmer AE, Reed JC. 2008. BI-1 regulates endoplasmic reticulum Ca2+ homeostasis downstream of Bcl-2 family proteins. The Journal of biological chemistry 283: 11477-11484.

Xu Q, Reed JC. 1998. Bax inhibitor-1, a mammalian apoptosis suppressor identified by functional screening in yeast. Molecular Cell 1: 337-346.

Zhang H, Huang Q, Ke N, Matsuyama S, Hammock B, Godzik A, Reed JC. 2000. Drosophila pro-apoptotic Bcl-2/Bax homologue reveals evolutionary conservation of cell death mechanisms. The Journal of biological chemistry 275: 27303-27306.

Zhu ZJ, Wu KC, Yung WH, Qian ZM, Ke Y. 2016. Differential interaction between iron and mutant alpha-synuclein causes distinctive Parkinsonian phenotypes in Drosophila. Biochimica et Biophysica Acta (BBA) - Bioenergetics 1862: 518-525.

PeerJ reviewing PDF | (2016:10:14014:2:0:NEW 6 Jan 2017) 
530 Figures Legends

\section{Figure 1 - Drosophila BI-1 has six TM domains that are evolutionarily conserved}

532 A) The Drosophila, human, mouse and mosquito homologues contain a BI-1 domain with the

533 Drosophila version situated between amino acids $21-223$ and the human version at $74-286$ as

534

535

536

537

538

539

540 determined by the NCBI Conserved Domain Database (Marchler-Bauer et al. 2015). They have six transmembrane-spanning regions as predicted by the Eukaryotic Linear Motif (ELM) (Dinkel et al. 2013) and TMpred (Artimo et al. 2012). It shows presence of a motif rich region, that contains a NES, ER retention motif, Atg8 and calmodulin binding motifs as identified using ELM. Sequence alignment was performed by Clustal Omega (Goujon et al. 2010; Sievers et al. 2011) and showed high conservation of the Bax inhibitor-1 domain (Hsap is Homo sapiens NP_001092046.1, Mmus is Mus musculus NP_001164506.1, Dmel is Drosophila melanogaster NP_648205.1 and Agam is Anopheles gambiae XP_315790.3). "*" indicate the residues that are identical, ":" indicate the conserved substitutions, "." indicate the semi-conserved substitutions.

543

544 Colours show the chemical nature of amino acids. Red is small hydrophobic (including aromatic), Blue is acidic, Magenta is basic, and Green is basic with hydroxyl or amine groups. B) Additional protein analysis performed using Phyre2 (Kelley et al. 2015) revealed the presence of seven transmembrane domains in both the Drosophila and human sequences (Image cartoons are obtained from Phyre2). C) The 3D modelling of the Drosophila and human proteins using Phyre 2 shows a close similarity in the structure and the orientation of the transmembrane domains with the image coloured by rainbow from the $\mathrm{N} \rightarrow \mathrm{C}$ terminus (Image cartoons are obtained from Phyre2).

Figure 2 - Reduction of $B I-1$ activity decreases survival and impairs climbing ability

A) The knockdown of $B I-1$ in the $D d c$-Gal4-expressing neurons results in reduced lifespan when compared to control flies expressing UAS-lacZ. The genotypes are Ddc-Gal4/UAS-lacZ, DdcGal4/UAS-BI-1-RNAi (1) and Ddc-Gal4/UAS-BI-1-RNAi (2). Longevity is shown as percent survival ( $\mathrm{P}<0.05$, determined by the log-rank (Mantel-Cox) test and $N \geq 200$ ). B) The knockdown of $B I-1$ in these neurons resulted in a significant decrease in climbing ability as determined by nonlinear fitting of the climbing curves and comparing the $95 \% \mathrm{CI}$. The genotypes are Ddc-Gal4/UAS-lacZ, Ddc-Gal4/ UAS-BI-1-RNAi (1) and Ddc-Gal4/UAS-BI-1$R N A i$ (2). Error bars indicate standard error of the mean and $N=50$.

A) The co-expression of Buffy with BI-1-RNAi in the Ddc-Gal4-expressing neurons result in the rescue of the observed phenotype of decreased survival when compared to the control. Genotypes are Ddc-Gal4/UAS-lacZ, UAS-Buffy; Ddc-Gal4/UAS-lacZ, UAS-Buffy; Ddc-Gal4/ $U A S-B I-1-R N A i$ (1), and UAS-Buffy; Ddc-Gal4/UAS-BI-1-RNAi (2). Longevity is shown as percent survival ( $\mathrm{P}<0.05$, determined by log-rank (Mantel-Cox) test with $N \leq 200)$. B) The knockdown of $B I-1$ along with the overexpression of Buffy in these neurons results in the suppression of the age-dependent loss in climbing ability. The genotypes are Ddc-Gal4/UASlacZ, UAS-Buffy; Ddc-Gal4/UAS-lacZ, UAS-Buffy; Ddc-Gal4/UAS-BI-1-RNAi (1), and UASBuffy; Ddc-Gal4/UAS-BI-1-RNAi (2). Analysis was done by nonlinear fitting of the climbing 
570 curves and significance was determined by comparing the $95 \% \mathrm{CI}$. Error bars indicate standard 571 error of the mean and $N=50$.

\section{Figure 4 - Knockdown of $\boldsymbol{B I}$ - 1 in neurons enhances the $\boldsymbol{\alpha}$-synuclein-induced phenotypes}

573

574

575

576

577

578

579

580

581

582

583

584

585

586

587

588

589

590

591

592

593

594

595

596

597

598

599

600

601

A) The directed knockdown of BI-1 along with $\alpha$-synuclein expression in the Ddc-Gal4expressing neurons resulted in a shortened lifespan when compared to the control. Genotypes are Ddc-Gal4/UAS-lacZ, UAS- $\alpha$-synuclein; Ddc-Gal4/UAS-lacZ, UAS- $\alpha$-synuclein; Ddc-Gal4/ UAS-BI-1-RNAi (1) and UAS- $\alpha$-Synuclein; Ddc-Gal4/UAS-BI-1-RNAi (2). Longevity is shown as percent survival $(\mathrm{P}<0.05$, determined by log-rank (Mantel-Cox) test with $N \leq 200)$. B) The coexpression of BI-1-RNAi with $\alpha$-synuclein resulted in a slight but significant decrease in the agedependent loss in climbing ability when compared to the control. The genotypes are Ddc-Gal4/ UAS-lacZ, UAS- $\alpha$-synuclein; Ddc-Gal4/ UAS-lacZ, UAS- $\alpha$-synuclein; Ddc-Gal4/UAS-BI-1$R N A i$ (1) and UAS- $\alpha$-synuclein; Ddc-Gal4/UAS-BI-1-RNAi (2). Analysis was done by nonlinear fitting of the climbing curves and significance was determined by comparing the $95 \% \mathrm{CI}$. Error bars indicate standard error of the mean and $N=50$.

\section{Figure 5 - Knockdown of $B I-1$ in the developing eye results in decreased ommatidia and} increased degeneration of the ommatidial array

Scanning electron micrographs when $B I-1$ is repressed in the Drosophila developing eye; (A) GMR-GAL4/UAS-lacZ, (B) GMR-GAL4/UAS-BI-1-RNAi (1) and (C) GMR-GAL4/UAS-BI-1$R N A i$ (2), when repressed along with overexpression of Buffy; D) UAS-Buffy; GMR-Gal4/UASlacZ, E) UAS-Buffy; GMR-Gal4/ UAS-BI-1-RNAi (1) and E) UAS-Buffy; GMR-Gal4/ UAS-BI-1$R N A i$ (2) and when co-expressed with $\alpha$-synuclein; G) UAS- $\alpha$-synuclein; GMR-Gal4/UAS-lacZ, H) UAS- $\alpha$-synuclein; GMR-Gal4/UAS-BI-1-RNAi, and I) UAS- $\alpha$-synuclein; GMR-Gal4/UAS$B I-1-R N A i$. J) Biometric analysis when $B I-1$ is repressed in the eye indicated decreased ommatidia number and higher percentage of ommatidial disruption when compared to the control. K) The co-expression of Buffy with both BI-1-RNAi lines resulted in the suppression of the eye phenotypes, the ommatidia number and disruption of the eye were restored to control levels. L) The knockdown of BI-1 along with $\alpha$-synuclein expression resulted in worsened eye phenotypes, the number of ommatidia was lower and the degree of ommatidial disruption was higher than either the knockdown of both BI- 1 lines or that of $\alpha$-synuclein when compared to controls. Comparisons were determined by one-way analysis of variance (ANOVA) with a Dunnett's multiple comparison post-test $(\mathrm{P}<0.05)$, error bars are standard error of the mean, $N=10$ and asterisks represent statistical significance $\left(* \mathrm{p}<0.05,{ }^{* *} \mathrm{p}<0.01\right.$ and $\left.* * * \mathrm{p}<0.001\right)$. 


\section{Figure 1}

Drosophila BI-1 has six TM domains that are evolutionarily conserved

A) The Drosophila, human, mouse and mosquito homologues contain a BI-1 domain with the Drosophila version situated between amino acids $21-223$ and the human version at 74 286 as determined by the NCBI Conserved Domain Database ( Marchler-Bauer et al. 2015 ) . They have six transmembrane-spanning regions as predicted by the Eukaryotic Linear Motif (ELM) ( Dinkel et al. 2013 ) and TMpred ( Artimo et al. 2012 ). It shows presence of a motif rich region, that contains a NES, ER retention motif, Atg8 and calmodulin binding motifs as identified using ELM. Sequence alignment was performed by Clustal Omega ( Goujon et al. 2010 ; Sievers et al. 2011 ) and showed high conservation of the Bax inhibitor-1 domain (Hsap is Homo sapiens NP_001092046.1, Mmus is Mus musculus NP_001164506.1, Dmel is Drosophila melanogaster NP_648205.1 and Agam is Anopheles gambiae XP_315790.3). "*" indicate the residues that are identical, ":" indicate the conserved substitutions, "." indicate the semi-conserved substitutions. Colours show the chemical nature of amino acids. Red is small hydrophobic (including aromatic), Blue is acidic, Magenta is basic, and Green is basic with hydroxyl or amine groups. B) Additional protein analysis performed using Phyre2 ( Kelley et al. 2015 ) revealed the presence of seven transmembrane domains in both the Drosophila and human sequences (Image cartoons are obtained from Phyre2). C) The 3D modelling of the Drosophila and human proteins using Phyre2 shows a close similarity in the structure and the orientation of the transmembrane domains with the image coloured by rainbow from the $\mathrm{N} \rightarrow \mathrm{C}$ terminus (Image cartoons are obtained from Phyre2). 
A.

MSHSSVTREAPQLLSQRQRREVRGWIUGCLPGPRGGPALFGLVTFGQSGDCCTDSGTMN 60

Mrap

Demel

Agam

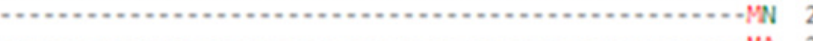

-

genes.

Hsap

Mmus

Denel

IFDRKIN - -FDAL - - LI FSHITPSTQQHL KKVYASFALCMFVAAAGAWWWTHFIQAGL IFDRKIN - FDAL - - LI FSHI TPSTQQHLKKVYASFALCMFVAGAGAWHWTHFIQAGL DTANYNDRFQTFMNGL GDRYEPYVREHLSKMMVGSTAAATANGAMLQM-RDFLDLGV MATSFANFSFERLSQQ GAKLDPRLRQHLSKYYGCLAATCSTATVGSLIHL - SGIWEAGL

$$
* *:
$$

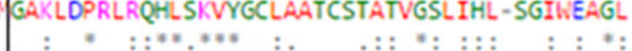

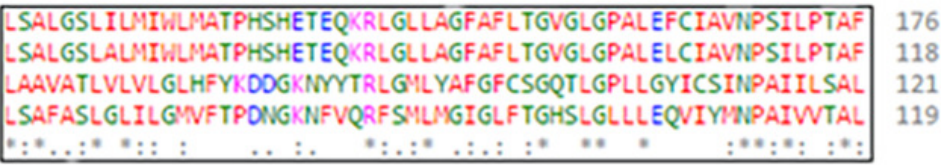

MGIAMIFTCFTLSALYARRRSYLFLGGILISALSLLLLSSLGWFFGSIWLFQANLYVGL 236 MGTAMIFTCFSLSALYARRRSYLFLGGILMSAMISUMLLSSLGNLFFGSIWLFQANLYLGL 178 TGTFVTFISLSLSALLAEQGKYLYLGGMLVSVINTMALLSLFWNFKSYFVQVTQLYVGV 181 VGITIIFACLTASAFFAKRGKYYYLGIUASALSTMALINLGNLFFRSYIVQOISLYLGL 179

Dere

Agam

Hsap

Nmus

Denel

Agam

I:TIZ RICH RIG:OX

WWCGFVLFDTQLIIEKAEHGDQDYINHCIDLFLDFITVFRKLPMILAWEEPKKKEKK - 295 LVMCGFVLFDTQLIIEKAEHGDKDYIWHCVDLFLDFVTLFRKLPLILAFNEX PKKKEKK - 237 FVMAAFIVDTQNIVEKCRNGNRDWQHALDLFFDVLSMFRRLLIILTQKEERKQNERRQ 241 IVMAGFVLFDTHMIMEKHRLGSNDFIGHSLDLFYOVISIF RRLLVILAQRECWERRKRK 239

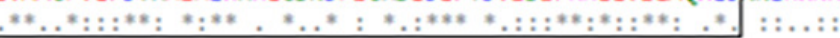

Hsap H...2 295

Mrus

295

NKTK 245

Denel

SN- - 241

B.
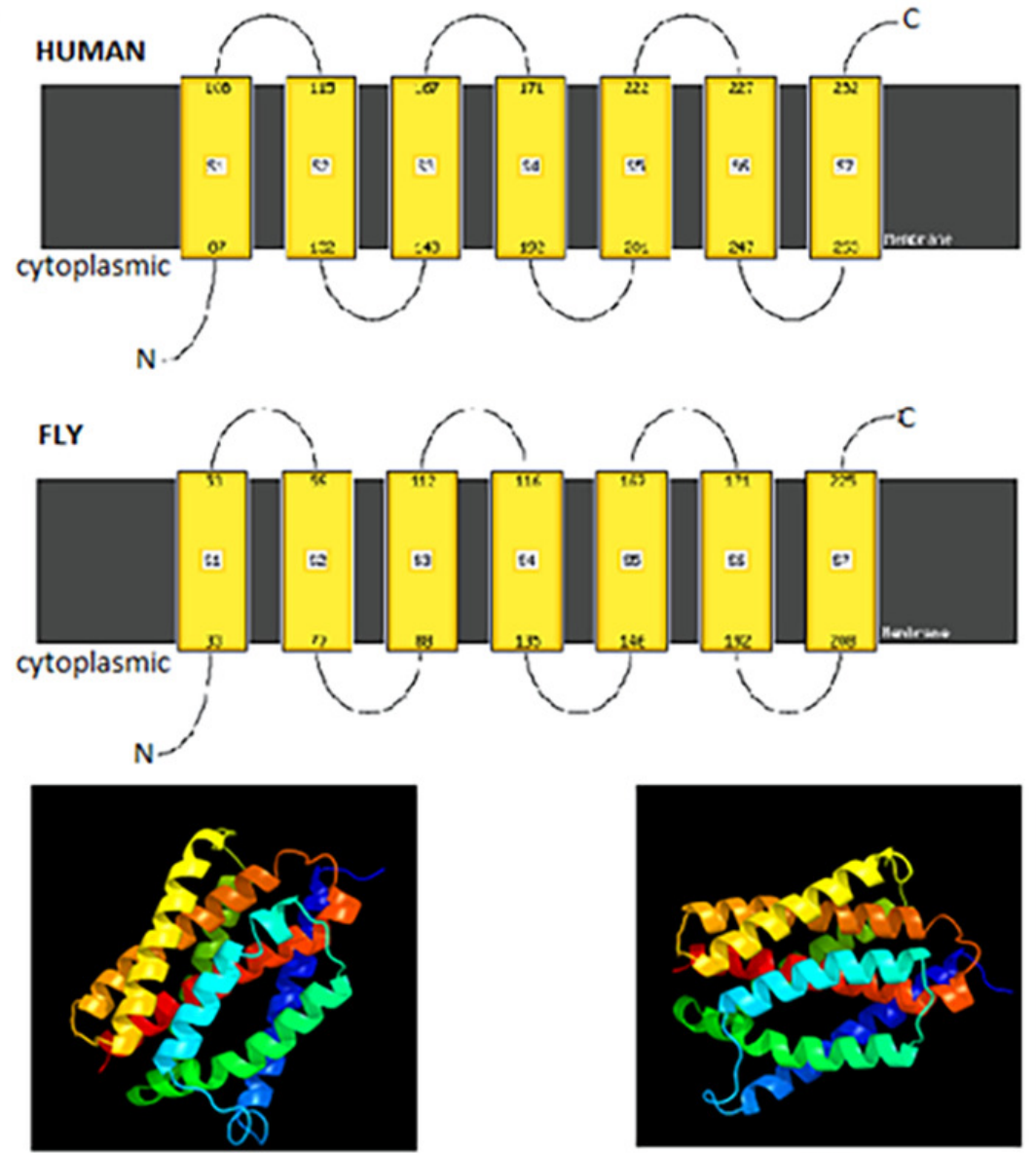

HUMAN
Peer) reviewing Pै̃
| (2016:10:14014:2:0:NEW 6 Jan 2017)

FLY 


\section{Figure 2 (on next page)}

Loss of BI-1 activity decreases survival and impairs climbing ability

A) The inhibition of $B I-1$ in the Ddc-Gal4-expressing neurons results in reduced lifespan when compared to control flies expressing the lacZ transgene. The genotypes are Ddc-Gal4/ UASlacZ, Ddc-Gal4/ UAS-BI-1-RNAi 1 and Ddc-Gal4/ UAS-BI-1-RNAi 2. Longevity is shown as percent survival ( $P<0.05$, determined by the log-rank (Mantel-Cox) test and $N \geq 200$ ). B) The inhibition of $\mathrm{Bl}-1$ in these neurons resulted in a significant decrease in climbing ability as determined by nonlinear fitting of the climbing curves and comparing the $95 \% \mathrm{Cl}$. The genotypes are Ddc-Gal4/ UAS-lacZ, Ddc-Gal4/ UAS-BI-1-RNAi 1 and Ddc-Gal4/ UAS-BI-1-RNAi 2. Error bars indicate standard error of the mean and $N=50$. 


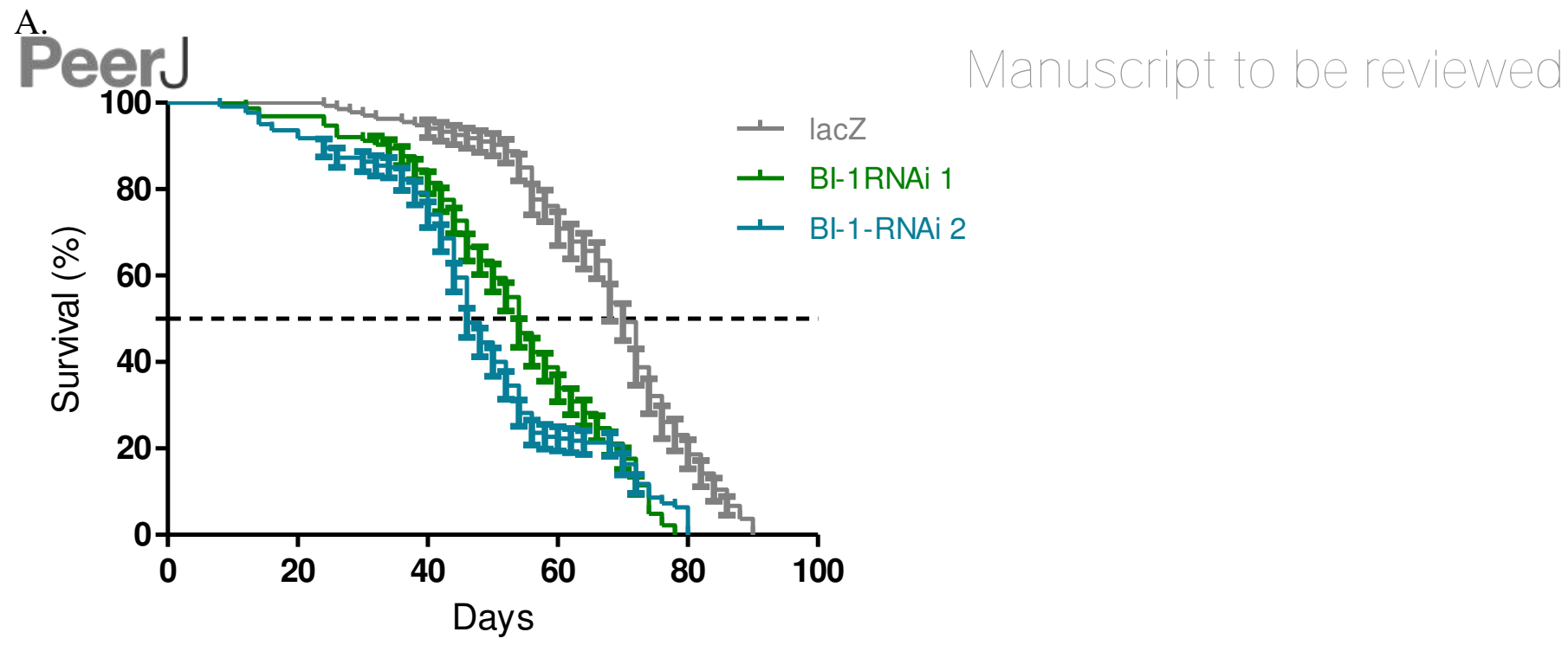

B.

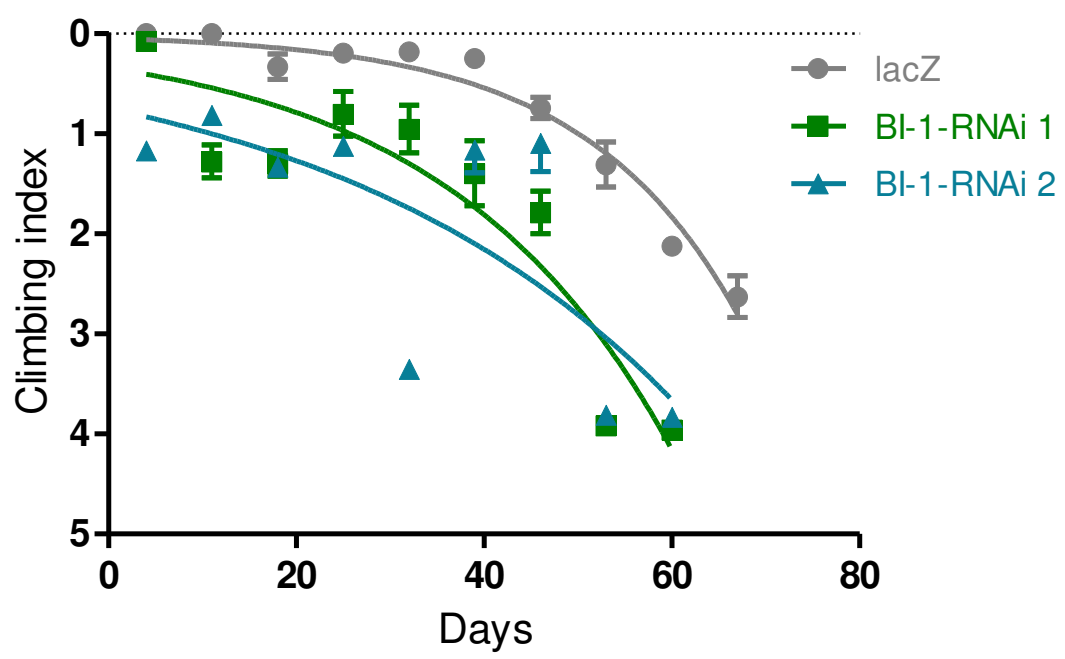




\section{Figure 3 (on next page)}

The BI-1-induced phenotypes can be suppressed by the overexpression of Buffy
A) The co-expression of Buffy with BI-1-RNAi in the Ddc-Gal4-expressing neurons result in the inhibition of the observed phenotype of decreased survival when compared to the control. Genotypes are UAS-Buffy; Ddc-Gal4/UAS-IacZ, UAS-Buffy; Ddc-Gal4/UAS-BI-1-RNAi 1, and UAS-Buffy; Ddc-Gal4/ UAS-BI-1-RNAi 2. Longevity is shown as percent survival $(\mathrm{P}<0.05$, determined by log-rank (Mantel-Cox) test with $N \leq 200$ ). B) The inhibition of $B I-1$ along with the overexpression of Buffy in these neurons results in the suppression of the age-dependent loss in climbing ability. The genotypes are UAS-Buffy; Ddc-Gal4/UAS-lacZ, UAS-Buffy; Ddc- Gal4/ UAS-BI-1-RNAi 1, and UAS-Buffy; Ddc-Gal4/ UAS-BI-1-RNAi 2. Analysis was done by nonlinear fitting of the climbing curves and significance was determined by comparing the $95 \% \mathrm{Cl}$. Error bars indicate standard error of the mean and $N=50$. 


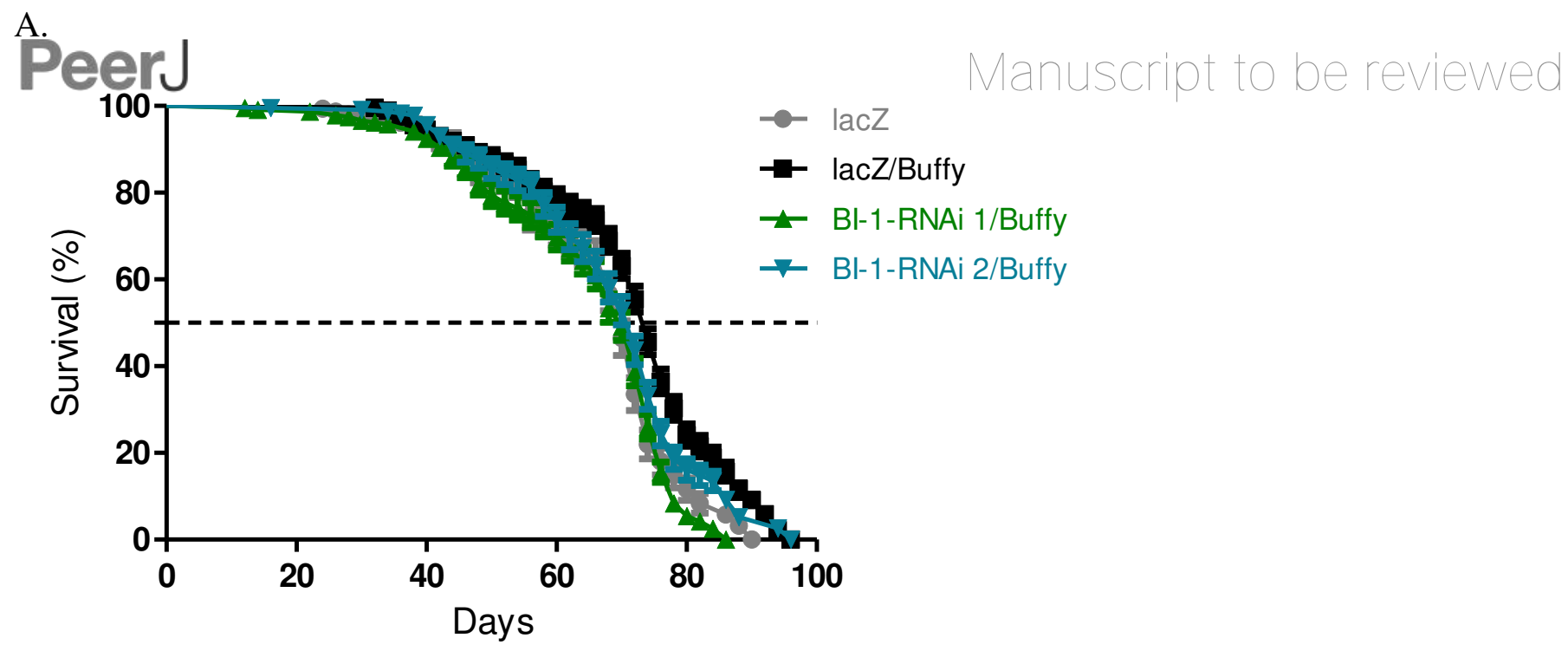

B.

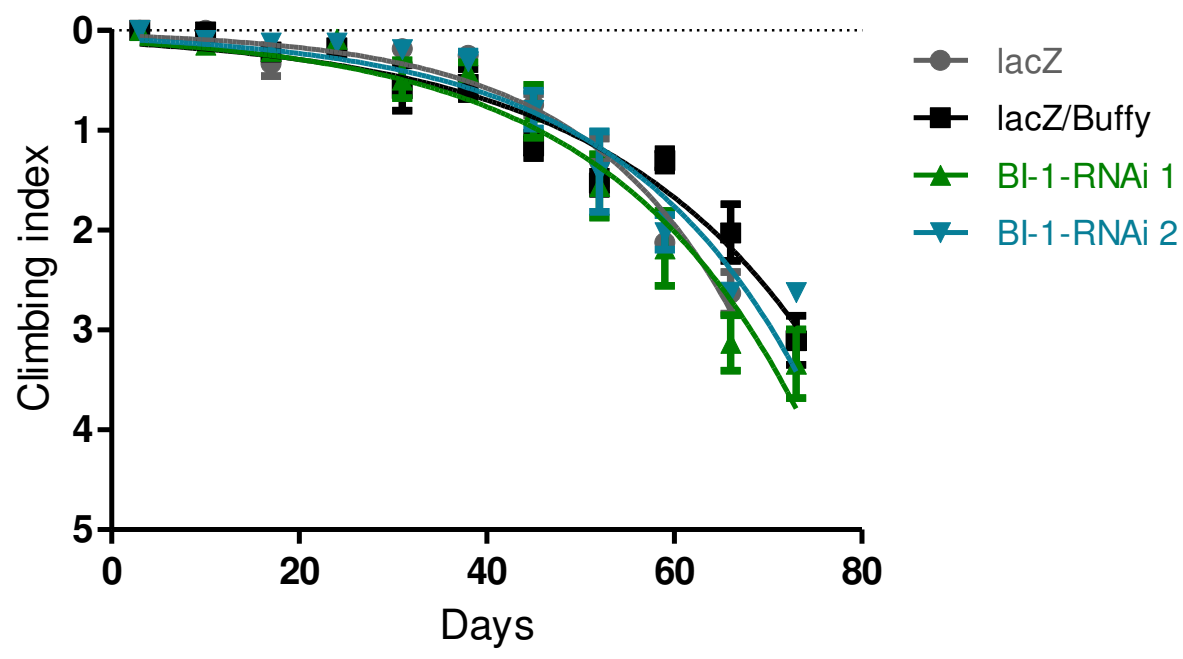




\section{Figure 4 (on next page)}

Loss of BI-1 in neurons complements the $\alpha$-synuclein-induced phenotypes
A) The inhibition of $B I-1$ along with $\alpha$-synuclein expression in the Ddc-Gal4-expressing neurons resulted in a shortened lifespan when compared to the control. Genotypes are UAS- $\alpha$-synuclein; Ddc-Gal4/ UAS-IacZ, UAS- $\alpha$-synuclein; Ddc-Gal4/ UAS-BI-1-RNAi 1 and UAS- $\alpha$ - synuclein; Ddc-Gal4/ UAS-BI-1-RNAi 2. Longevity is shown as percent survival $(\mathrm{P}<0.05$, determined by log-rank (Mantel-Cox) test with $N \leq 200$ ). B) The co-expression of BI-1-RNAi with $\alpha$-synuclein resulted in a slight but significant decrease in the age-dependent loss in climbing ability when compared to the control. The genotypes are UAS- $\alpha$-synuclein; Ddc- Gal4/ UAS-IacZ, UAS- $\alpha$-synuclein; Ddc-Gal4/ UAS-BI-1-RNAi 1 and UAS- $\alpha$-synuclein; Ddc-Gal4/ UAS-BI-1-RNAi 2. Analysis was done by nonlinear fitting of the climbing curves and significance was determined by comparing the $95 \% \mathrm{Cl}$. Error bars indicate standard error of the mean and $N=50$. 


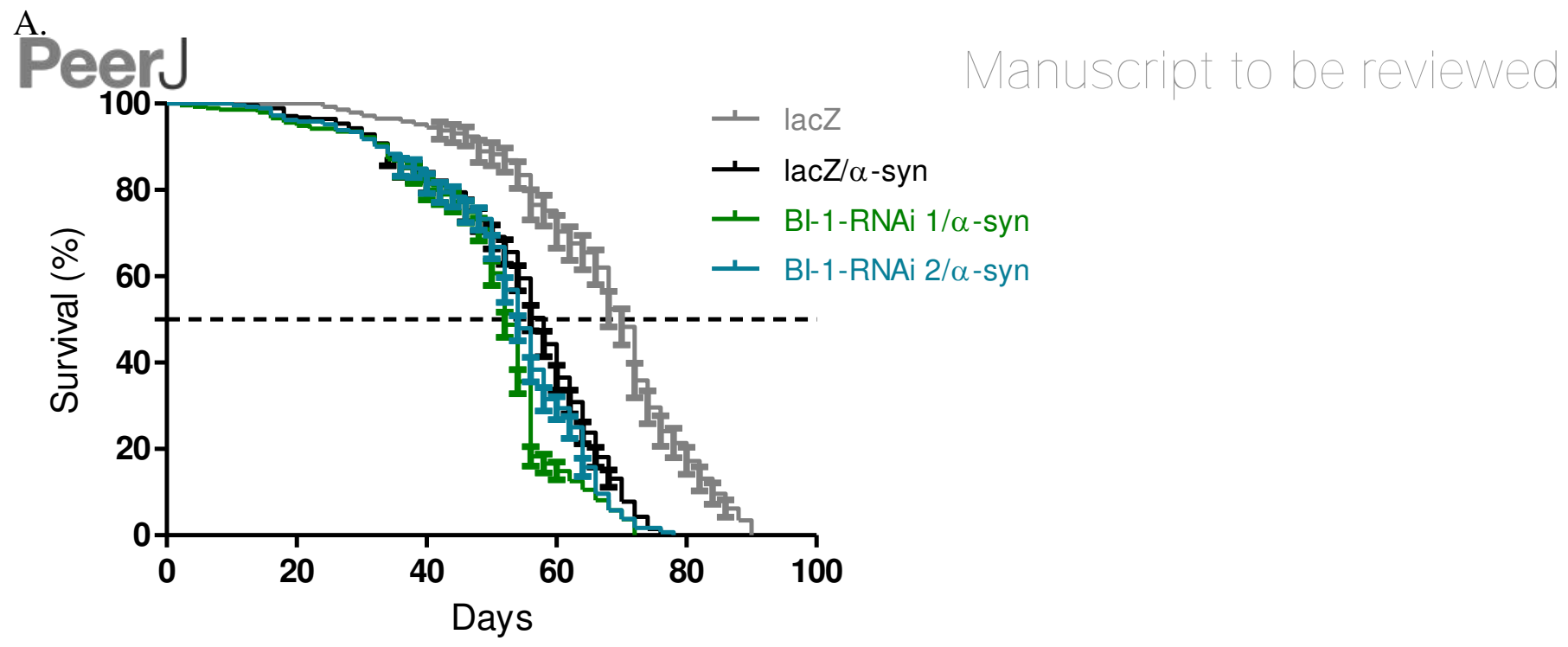

B.

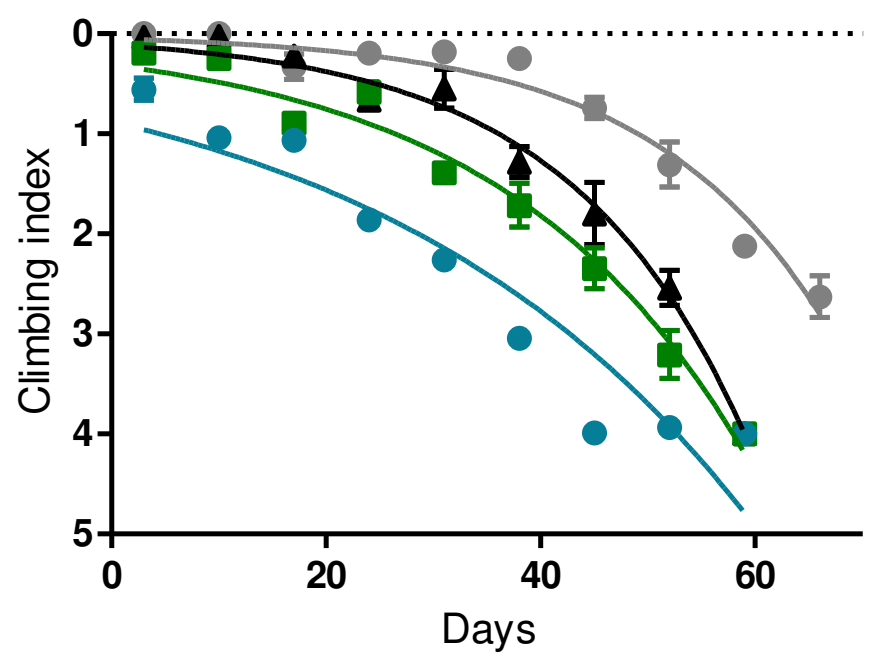

- lacZ

$\leftarrow$ lacZ/ $\alpha-$ syn

t- BI-1-RNAi 2/ $\alpha$-syn

$\rightarrow$ BI-1-RNAi 2/ $\alpha$-syn 


\section{Figure 5}

Knockdown of $\mathrm{BI}-1$ in the developing eye results in decreased ommatidia and increased degeneration of the ommatidial array

Scanning electron micrographs when $B I-1$ is repressed in the Drosophila developing eye; (A) GMR-GAL4/ UAS-IacZ, (B) GMR-GAL4/ UAS-BI-1-RNAi (1) and (C) GMR-GAL4/ UAS-BI-1-RNAi (2), when repressed along with overexpression of Buffy; D) UAS-Buffy; GMR-Gal4/ UAS-lacZ, E) UAS-Buffy; GMR-Gal4/ UAS-BI-1-RNAi (1) and E) UAS-Buffy; GMR-Gal4/ UAS-BI-1-RNAi (2) and when co-expressed with $\alpha$-synuclein; G) UAS- $\alpha$-synuclein; GMR-Gal4/ UAS-lacZ, H) UAS$\alpha$-synuclein; GMR-Gal4/ UAS-BI-1-RNAi, and I) UAS- $\alpha$-synuclein; GMR-Gal4/ UAS-BI-1-RNAi. J) Biometric analysis when $B I-1$ is repressed in the eye indicated decreased ommatidia number and higher percentage of ommatidial disruption when compared to the control. K) The coexpression of Buffy with both BI-1-RNAi lines resulted in the suppression of the eye phenotypes, the ommatidia number and disruption of the eye were restored to control levels.

L) The knockdown of $B \mathrm{I}-1$ along with $\alpha$-synuclein expression resulted in worsened eye phenotypes, the number of ommatidia was lower and the degree of ommatidial disruption was higher than either the knockdown of both BI-1 lines or that of $\alpha$-synuclein when compared to controls. Comparisons were determined by one-way analysis of variance (ANOVA) with a Dunnett's multiple comparison post-test $(P<0.05)$, error bars are standard error of the mean, $N=10$ and asterisks represent statistical significance $(* p<0.05, * * p<0.01$ and $\left.{ }^{* * *} p<0.001\right)$. 

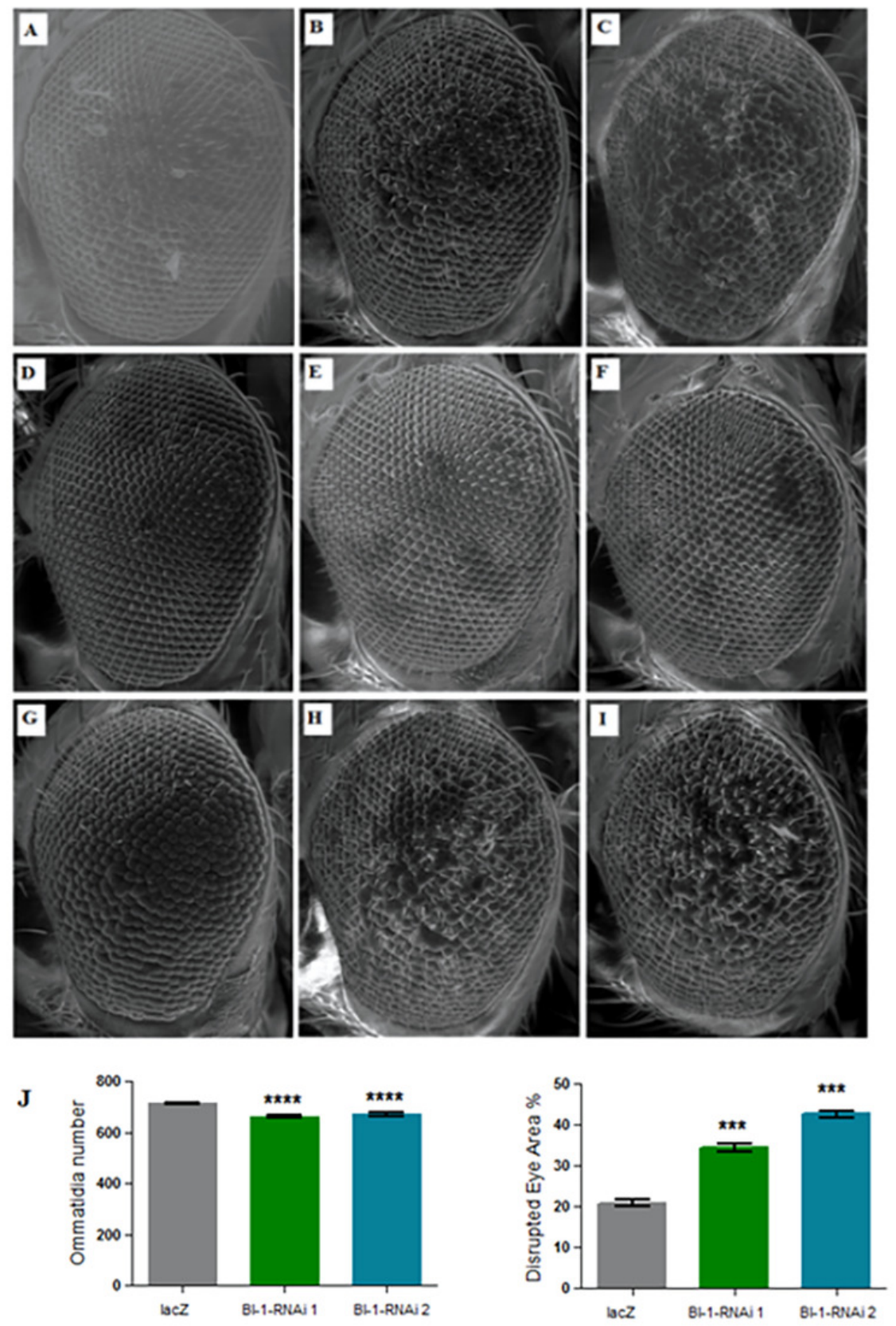

K
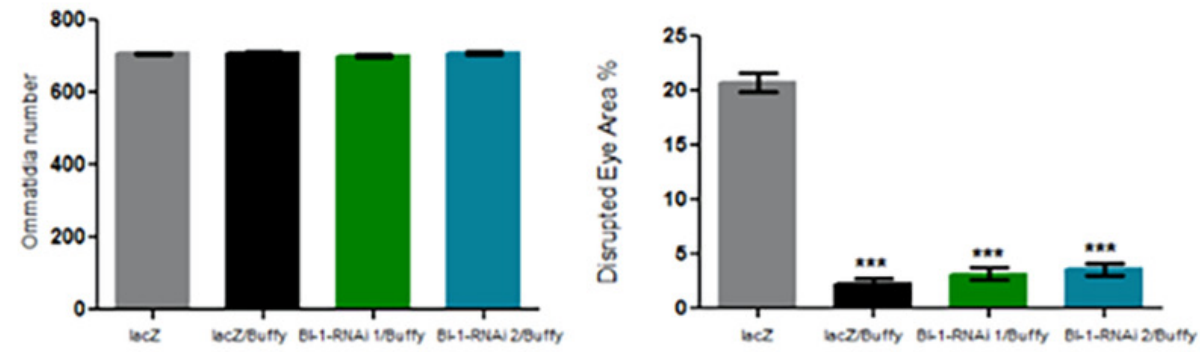

$\mathbf{L}$
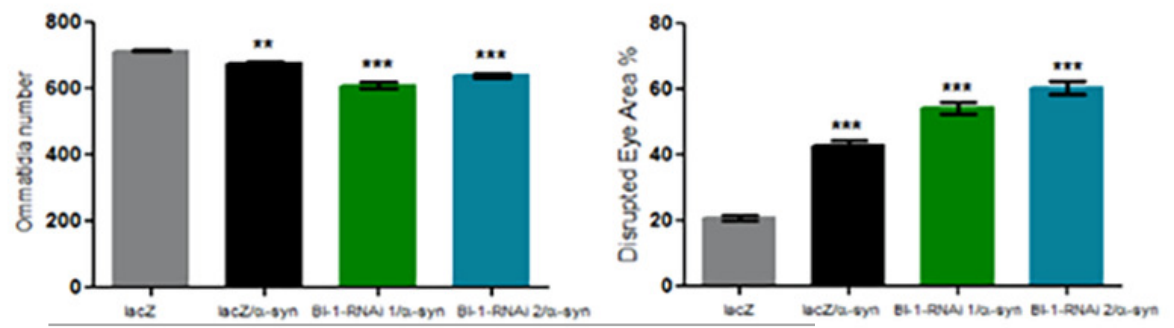

PeerJ reviewing PDF | (2016:10:14014:2:0:NEW 6 Jan 2017) 